\title{
Spectral Analysis of Translation-Invariant Mechanical Systems with Application to Structural Vibrations and Stability
}

\author{
${ }^{1}$ Nikolay V. Banichuk, ${ }^{2}$ Alexander A. Barsuk, ${ }^{1}$ Svetlana Yu. Ivanova, ${ }^{3}$ Tero T. Tuovinen \\ ${ }^{1}$ Ishlinsky Institute for Problems in Mechanics RAS, Moscow, 119526, Russia \\ ${ }^{2}$ State University of Moldova, Chișinău, Moldova \\ ${ }^{3}$ JAMK University of Applied Sciences Jyväskylä, Jyväskylä, Finland
}

Received: April 17, 2021. Revised: October 21, 2021. Accepted: November 18, 2021. Published: December 6, 2021.

\begin{abstract}
The paper considers the problems and the methods of spectral analysis of elastic structural systems. The presented consideration focuses on the translation-invariant spectral formulations. Some periodic representations and the spectral decomposition are derived. In the context of general analysis of translation-invariant systems, the particular problems of structural vibration and stability are solved in analytical form.
\end{abstract}

Keywords: Mechanical Systems, Spectral Analysis, Structural Vibrations, Stability

\section{INTRODUCTION}

The behavior of mechanical structure that is loaded by external forces is completely characterized by its frequency spectrum and corresponding modes of free vibrations. As for structural stability, the spectrum of critical power parameters and buckling shapes determine instability phenomenon. In the class of general spectrum problems including the eigenvalue problems of the theories of vibrations and stability, the important place is assigned to the corresponding spectral boundary-value problems of translation-invariant structures.

By definition, the translation-invariant systems are infinitely extensive and can be considered as asymptotic models of the real objects. Analysis and computation are appreciably simplified because of the absence of length parameter in these models. In addition, the qualitative and quantitative characteristics are presented and, in many cases, the corresponding problems solutions can be found in closed analytical form.

The present paper focuses on the fundamental questions of spectrum analysis such as convenient representations and effective decomposition, definition of the special invariance of the problems operators, required boundary conditions for the obtained elementary cells. Special attention is devoted to the important examples of harmonic vibrations and stability of one-dimensional and two-dimensional translation-invariant mechanical structures. These examples are useful for clear explanation of the obtaining and formation of the eigenvalue spectrum of harmonic frequencies and critical buckling loads.

We mention here the analytical approaches to translationinvariant systems developed in the monographs [1 -4$]$ and in the papers [5-11].

Note that the problems of harmonic vibrations and elastic stability of unbounded structural elements (strings, beams, plates) lying on the rigid end elastic supports belong to the class of periodic spectral problems defined at the infinite domains and having the analogies in many divisions of physics. As an example, we shall indicate the problem of solid physics - the problem of determination of the electron energetic spectrum in the periodic potential field, considered for the first time in [12].

Note also that the discussions as for theoretical aspects of the solutions of periodic spectral problems as multiple applications are contained in monographs [ $1-4]$.

\section{TRANSLATION-INVARIANT SYSTEMS}

To describe the translation-invariant system, we introduce Cartesian coordinates and orient the $x$-axis along the direction of translation symmetry. Consider the boundaryvalue problem defining the eigenvalues and eigenfunctions

$$
A w(q, x)=\lambda B w(q, x), \quad x \in(-\infty, \infty)
$$

where the operators $A$ and $B$ are invariant with respect to the shift operation

$$
x \rightarrow x+k a
$$

and include some boundary conditions, i.e., $A$ and $B$ are not changed, when the argument $x$ is replaced: $x \rightarrow x+k a$. Here and in what follows, the real value $a$ and integers $k, n$ are connected by the relations

$$
a=\frac{2 \pi}{n}, \alpha_{\mathrm{k}}=\frac{2 \pi}{n}(k-1)=\alpha a, k \in[1, n] \text {. }
$$

Denote by $\lambda$ and $w(q, x)$, respectively, the eigenvalue and the corresponding eigenfunction of the boundary-value problem (1). The collection of some important parameters, different from $x$, are denoted by $q$. Consider at first the case, when $\lambda$ is a simple eigenvalue and $w(q, x)$ is unique to within arbitrary constant. Define the function $\tilde{w}(q, x) \equiv$ $w(q, x+a)$. If we substitute $\tilde{w}(q, x)$ in (1) and fulfill the replacement $x+a \rightarrow x$ taking into account the translation-invariant property of the operators $A$ and $B$, 


$$
\tilde{w}(q, x)
$$

then we obtain that the function

is also the eigenfunction oれthe boundary-value problem (1) belonging to the eigenvalue . Since is simple, then

$$
\begin{aligned}
& \tilde{w}(q, x) \equiv C w(q, x), \\
& w(q, x+a) \equiv C w(q, x)
\end{aligned}
$$

where $C$ is some arbitrary constant. The eigenfunction property (4) shows that to find the eigenfunctions corresponding to single eigenvalues, it is sufficient to consider the interval $x \in[0, a]$. In accordance with (4), the values of functions in the interval $[q, 2 \pi] x)^{\text {are obtained as an }}$ extension of the function from the interval

$[0, a]$. In particular

$$
\begin{aligned}
& w(q, x+2 a) \equiv C w(q, x+a) \equiv C^{2} w(q, x), \\
& w(q, x+k a) \equiv C^{k} w(q, x) .
\end{aligned}
$$

Since $n a=2 \pi$, then

$$
w(q, x+a) \equiv C^{n} w(q, x) \equiv w(q, x) .
$$

Thus, the constant $C$ in (5) satisfies the following equation:

$$
C^{n}=1 \text {. }
$$

If we denote the roots of the equation (6) by

$$
\gamma_{n}^{k}=\exp \left[i \frac{2 \pi}{n}(k-1)\right], k=1,2, \ldots, n,
$$

then, the second relation in (4) can be represented as

$$
w_{k}(q, x+a)=\gamma_{n}^{k} w_{k}(q, x)
$$

where $i$ is an imaginary unit and $k=1,2, \ldots, n$.

Consider now the case of the multiple eigenvalue with multiplicity $r$ and corresponding system of linearly independent eigenfunctions $w_{1}(q, x), \ldots, w_{r}(q, x)$ of the boundary-value problem (1). Suppose that the system of these functions satisfies the normalization conditions

$$
\left(w_{k}(q, x), B w_{l}(q, x)\right)=\delta_{k l}, \quad k, l=1,2, \ldots, r .
$$

$$
\text { The functions } \quad \tilde{w}_{j}(q, x) \equiv w_{j}(q, x+a)
$$

( $j=1,2, \ldots, r)$ satisfy the boundary-value problem (1) and, as it follows from the translation-invariance property of operator $B$ with respect to shift $x \rightarrow x+a$, the orthonormalization condition (9) is also satisfied for the system of functions $\tilde{w}_{j}(q, x)$. Using the system of eigenfunctions $w_{j}(q, x)$, the functions $\tilde{w}_{j}(q, x)$ can be represented in the form of expansions

$$
\tilde{w}_{k}(q, x)=\sum_{l=1}^{r} G_{k l} w_{l}(q, x)
$$

with coefficients $G_{k l}$. Substituting the expansions (10) into orthonormalization conditions (9) and taking into account the properties of the eigenfunctions $w_{j}(q, x)$, we will have

$$
\sum_{s=1}^{r} G_{k s}^{*} G_{l s}=\delta_{k l}
$$

The expansion coefficients $G_{l s}$ constitute the unitary matrix, i.e., $\tilde{G}^{*} G=I$, where $I$ is an unit matrix. The matrix $G$ can be reduced to diagonal form using the transformation matrix $U$ constituted from eigenfunctions $w_{j}(q, x)$ and satisfying the orthonormalization conditions (9), i.e.

$$
U^{*} G U=\Lambda, \Lambda_{j k}=\kappa_{j} \delta_{j k} .
$$

Thus, the eigenfunction corresponding the multiple eigenvalue of the invariant (with respect to shift $x \rightarrow x+a$ ) boundary-value problem (1) can be taken in the form satisfying the relations (4), i.e.

$$
w_{l}(q, x+a) \equiv C_{l} w_{l}(q, x), \quad l=1,2, \ldots, r .
$$

By analogy with the case of simple eigenvalues, we can derive the general property of the eigenfunctions of the translationinvariant boundary-value problem as

$$
w_{\alpha}(q, x+a) \equiv \exp (i \alpha a) w_{\alpha}(q, x) .
$$

\section{SOME REPRESENTATIONS AND DECOMPOSITION}

Consider the function $w_{\alpha}(q, x)$ from (14) and introduce the function $w_{\alpha}^{0}(q, x)$ as

$$
\begin{aligned}
& w_{\alpha}^{0}(q, x) \equiv \exp \left(-\frac{i \alpha_{k} x}{a}\right) w_{\alpha}(q, x)= \\
& =\exp [-i(k-1) x] w_{\alpha}(q, x) .
\end{aligned}
$$

Consider also the value of this function when $x \rightarrow x+a$. We will have

$$
\begin{aligned}
& w_{\alpha}^{0}(q, x+a) \equiv \\
& \equiv \exp \left(-\frac{i \alpha_{k} x}{a}\right) \exp \left(-i \alpha_{k}\right) w_{\alpha}(q, x+a)= \\
& =w_{\alpha}^{0}(q, x) .
\end{aligned}
$$


Thus, the function $w_{\alpha}^{0}(q, x)$ is periodic with period $a$. As a result of conducted analysis of translation-invariant system, we have

$$
\begin{aligned}
& w_{\alpha}(q, x) \equiv \exp \left(\frac{i \alpha_{k} x}{a}\right) w_{\alpha}^{0}(q, x)= \\
& =\exp [-i(k-1) x] w_{\alpha}^{0}(q, x), \\
& w_{\alpha}^{0}(q, x+a) \equiv w_{\alpha}^{0}(q, x), \\
& k=1,2, \ldots, n, \quad 0 \leq \alpha a \leq 2 \pi, \\
& \lambda(\alpha a)=\lambda(2 \pi-\alpha a) .
\end{aligned}
$$

The solution of the boundary-value problem (1) defined at $x \in(-\infty,+\infty)$ is replaced by the solution depending on the parameter $\alpha a \in[0,2 \pi]$ at the period $x \in[0, a]$ (method of decomposition for translation-invariant problems):

$$
\begin{aligned}
& A w(q, x)=\lambda w B(q, x), \quad 0 \leq \alpha a \leq 2 \pi, \\
& w^{(s)}(q, a)=\exp (i \alpha a) w^{(s)}(q, 0), \\
& s=0,1,2 \ldots .
\end{aligned}
$$

The maximal order of derivative is coordinated with the operators $A$ and $B$.

It should be noted the variational formulation of the boundary-value problem (17)

$$
\begin{aligned}
& \lambda=\min _{w}\left\{\frac{(w, A w)_{\Omega_{0}}}{(w, B w)_{\Omega_{0}}}\right\}, \quad 0 \leq \alpha a \leq 2 \pi, \\
& w^{(s)}(q, a)=\exp (i \alpha a) w^{(s)}(q, 0), \\
& s=0,1,2 \ldots .
\end{aligned}
$$

It is important that the formulation of the spectral boundaryvalue problem invariant with respect to finite shift at elementary cell $\Omega_{0} \quad(0<x<a)$ is correct as for scalar as vector state function.

Using the relations (16), we emphasize that in the context of numerical realization of (17) or (18) it is enough to carry out all operations at $\alpha a \in[0, \pi]$.

Let $\lambda_{1}(\alpha a), \lambda_{2}(\alpha a), \ldots$ are eigenvalues of the problem (17) for fixed value of the parameter $\alpha a$. The values $\lambda_{i}(\alpha a)$ are the continuous functions in the case of continuous variation of the argument. Denote by $\left(\lambda_{i}\right)_{\min }$ and $\left(\lambda_{i}\right)_{\max }$ the minimum and maximum values of these functions. If $\left(\lambda_{i}\right)_{\max }<\left(\lambda_{i+1}\right)_{\min }$ for all $i$, then we conclude that the spectrum of eigenvalues of the considered translation-invariant problems consists of nonoverlapping intervals with continuous spectrum. Note that the domains with continuous spectrum are transformed into "condensed zones" in the case of extensive (lengthy) systems.

\section{APPLICATION OF SPECTRAL ANALYSIS TO MECHANICAL SYSTEMS}

In what follows, we will present the application of the translation-invariant problems of structural vibration and stability.

\section{A. Harmonic Vibrations of Continuous String with Periodic Supports and Unbounded Length}

Let the continuous string with tension $T$, density $\rho$, cross-section area $S$, and elastic supports at the points $x_{j}=j l(j=0, \pm 1, \pm 2, \ldots)$ is described by the spectral boundary-value problem for the amplitude transverse vibration function $w(x)$

$$
\begin{aligned}
& \frac{d^{2} w(x)}{d x^{2}}+\omega^{2} w(x)=0, \quad-\infty<x<\infty \\
& \left(\frac{d w}{d x}\left(x_{j}\right)\right)^{+}-\left(\frac{d w}{d x}\left(x_{j}\right)\right)^{-}-\gamma w\left(x_{j}\right)=0, \\
& x_{j}=0, \pm 1, \pm 2 \ldots
\end{aligned}
$$

written in variables $\tilde{x}=x / l, \quad \tilde{\omega}^{2}=\rho S \omega^{2} l^{2} / T$, $\tilde{\gamma}=l \gamma / T$, where $\omega$ is frequency and $\gamma$ is rigidity of elastic supports (in what follows the tilde is omitted). The boundary-value problem (19) is translation invariant with respect to shift ( $j$ is an arbitrary integer)

$$
x \rightarrow x+j l
$$

and its analysis is reduced to the elementary interval $(0,1)$ as

$$
\begin{aligned}
& \frac{d^{2} w(x)}{d x^{2}}+\omega^{2} w(x)=0, \quad 0<x<1, \\
& 0 \leq \alpha \leq 2 \pi \\
& w(1)=e^{i \alpha} w(0), \\
& \left(\frac{d w}{d x}(0)\right)^{+}-e^{-i \alpha}\left(\frac{d w}{d x}(1)\right)^{-}-\gamma w(0)=0 .
\end{aligned}
$$

General solution and boundary conditions of (20) lead to the relation 


$$
\cos \alpha=\cos \omega+\frac{\gamma}{2 \omega} \sin \omega \equiv f(\omega) .
$$

Taking into account that $|\cos \alpha| \leq 1$ and consequently $|f(\omega)| \leq 1$, we obtain that the free string vibrations are realized with the frequencies filling the intervals with the boundaries $(f(\omega)= \pm 1)$ :

$$
\begin{aligned}
& f(\omega)=1: \quad \sin \frac{\omega}{2}=0, \quad \tan \frac{\omega}{2}=\gamma \frac{\omega}{2}, \\
& f(\omega)=-1: \quad \cos \frac{\omega}{2}=0, \quad \cot \frac{\omega}{2}=-\gamma \frac{\omega}{2} .
\end{aligned}
$$

The frequencies satisfying conditions $\sin (\omega / 2)=0$, $\cos (\omega / 2)=0$ determine the lower bounds of the bands with continuous spectrum and correspond to the harmonic vibrations of the string with free ends. The conditions $\tan (\omega / 2)=\gamma \omega / 2 \quad, \quad \cot (\omega / 2)=-\gamma \omega / 2$ determine the upper bounds of the frequency bands corresponding the string harmonic vibrations with elastic clamping (of rigidity $\gamma$ ) at the ends. Thus, the frequency spectrum of unbounded string with periodic elastic supports consists of the bands with continuous spectrum, which boundaries are determined by the conditions (22).

B. Free Transverse Vibrations of Unbounded ContinuousSolid Beam Lying on Supports with Equal Spans

Periodic translation-invariant boundary-value problem of transverse vibrations of the beam (Fig. 1) with bending rigidity $D$, cross-section area $S$, density $\rho$ is lying on the supports at the points $x_{j}=j l(j=0, \pm 1, \pm 2, \ldots)$ and admit the shift: $x \rightarrow x+j l$. Using the notation $\tilde{x}=x / l, \rho S \omega^{2} l^{4} / D=\tilde{\omega}^{2}$ (the tilde is omitted), the considered spectral problem can be formulated on elementary cell as

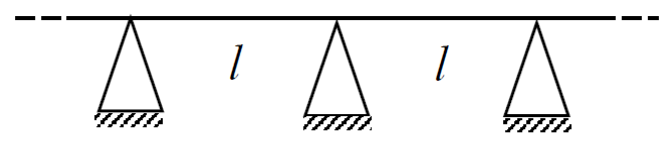

Fig. 1 Beam with equal spans.

$$
\begin{aligned}
& \frac{d^{4} w(x)}{d x^{4}}-\omega^{2} w(x)=0, \quad \Omega_{0}: 0<x<1, \\
& w(0)=0, \quad w(1)=0, \\
& \frac{d w}{d x}(1)=e^{i \alpha} \frac{d w}{d x}(0), \\
& \frac{d^{2} w}{d x^{2}}(1)=e^{i \alpha} \frac{d^{2} w}{d x^{2}}(0) .
\end{aligned}
$$

Using the general solution of the differential equation in (23)

$$
\begin{aligned}
& w(x)=c_{1} \cosh (\sqrt{\omega} x)+c_{2} \cos (\sqrt{\omega} x)+ \\
& +c_{3} \sinh (\sqrt{\omega} x)+c_{4} \sin (\sqrt{\omega} x)
\end{aligned}
$$

and the corresponding boundary conditions used for determination of the arbitrary constants $c_{1}, c_{2}, c_{3}, c_{4}$, we can derive the frequency equation

$\cos \alpha=$

$$
\begin{aligned}
& =\frac{\sinh \sqrt{\omega} \cos \sqrt{\omega}-\cosh \sqrt{\omega} \sin \sqrt{\omega}}{\sinh \sqrt{\omega}-\sin \sqrt{\omega}} \equiv \\
& \equiv f(\omega) .
\end{aligned}
$$

Since $|\cos \alpha| \leq 1$, then $|f(\omega)| \leq 1$. If $\omega \square 1$, then the asymptotic behavior of the function $f(\omega)$ from (24) is

$$
f(\omega) \approx \cos \sqrt{\omega}-\sin \sqrt{\omega} .
$$

The lower and the upper frequency bounds denoted by $\omega_{\min }$ and $\omega_{\max }$ are determined with the help of the equation $|f(\omega)|=1$. We will have

$$
\begin{aligned}
& f(\omega)=-1: \\
& \cos \frac{\sqrt{\omega}}{2}=0, \quad \tan \frac{\sqrt{\omega}}{2}=\tanh \frac{\sqrt{\omega}}{2}, \\
& f(\omega)=1: \\
& \sin \frac{\sqrt{\omega}}{2}=0, \quad \tan \frac{\sqrt{\omega}}{2}=-\tanh \frac{\sqrt{\omega}}{2} .
\end{aligned}
$$

Analysis of the solution (25) shows that the lower boundaries of bands with continuous frequency spectrum correspond to vibration of simply supported beam $(\cos (\sqrt{\omega} / 2)=0$, 
$\sin (\sqrt{\omega} / 2)=0$ ). The upper band boundaries correspond to the rigidly clamped beam at both ends ( $\tan (\sqrt{\omega} / 2)= \pm \tanh (\sqrt{\omega} / 2)$ ). Note that for bounded continuous beam with length $L$ lying on the periodic supports with equal spans $l$, the continuous spectrum is transformed into "condensed spectrum" when $L / l \square 1$.

\section{Stability of Unbounded Continuous Beam under} Compression

Consider the unbounded beam (Fig. 2) lying on the system of supports at the points $x_{j}=j l(j=0, \pm 1, \pm 2, \ldots)$ with equal spans of length $l$. The beam having the bending rigidity $D$ is under compression by longitudinal force $P$ and admit the shift $x \rightarrow x+j l$. The described translationinvariant system can be formulated at the elementary cell $\Omega_{0}$ as

$$
\begin{aligned}
& \frac{d^{4} w(x)}{d x^{4}}+\lambda \frac{d^{2} w(x)}{d x^{2}}=0, \\
& \Omega_{0}: 0<x<1, \quad 0 \leq \alpha \leq 2 \pi
\end{aligned}
$$

with boundary conditions from (23), which can be used for determination of the integration constants $d_{j}$ ( $j=1,2,3,4)$ in the general solution

$w(x)=d_{1} \cos (\sqrt{\lambda} x)+d_{2} \sin (\sqrt{\lambda} x)+d_{3} x+d_{4}$.

Using the notations $\tilde{x}=x / l, \lambda=P l^{2} / D$ (the tilde is omitted) and standard operation, we derive the following equation:

$$
\cos \alpha=\frac{\sqrt{\lambda} \cos \sqrt{\lambda}-\sin \sqrt{\lambda}}{\sqrt{\lambda}-\sin \sqrt{\lambda}} \equiv f(\lambda) .
$$

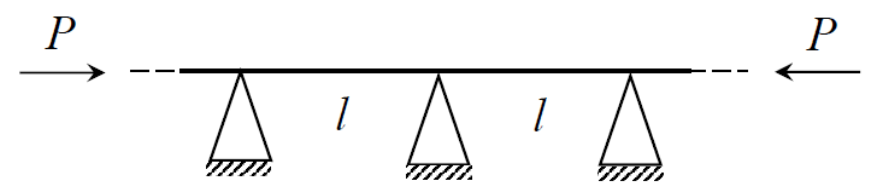

Fig. 2 Beam under compression.
Taking into account the inequality $|\cos \alpha| \leq 1$ and consequently $|f(\lambda)| \leq 1$, we conclude that the spectrum of critical buckling forces consists of the bands with continuous spectrum. As it follows from (27), the bands boundaries are determined with the help of the equation $f(\lambda)= \pm 1$ and have the form

$$
\begin{gathered}
f(\lambda)=-1: \quad \sqrt{\lambda}(1+\cos \sqrt{\lambda})=2 \sin \sqrt{\lambda}, \\
f(\lambda)=1: \sqrt{\lambda}(\cos \sqrt{\lambda}-1)=0 .
\end{gathered}
$$

Represented equality (28) is satisfied by the roots of equations

$$
\cos (\sqrt{\lambda} / 2)=0, \quad \tan (\sqrt{\lambda} / 2)=\sqrt{\lambda} / 2 .
$$

The equation (29) has solution $\sqrt{\lambda_{k}}=2 \pi k, k \neq 0$. The solution $\lambda=0$ is excluded from the considerations. In addition, the function $f(\lambda)$ achieves the maximum value $f(\lambda)=1$ for $\sqrt{\lambda_{k}}=2 \pi k$. The roots of the equation $\cos (\sqrt{\lambda} / 2)=0$ are given by the formula $\sqrt{\lambda_{k}}=(2 k+1) \pi$ and the roots of the equation $\tan (\sqrt{\lambda} / 2)=\sqrt{\lambda} / 2$ are represented as $\sqrt{\lambda_{k}}=(2 k+1) \pi-2 \varepsilon_{k} \quad(k=1,2, \ldots)$, where $\varepsilon_{k} \approx 2 /(2 k+1) \pi$ when $k \square 1$. Note that the asymptotic value of $\varepsilon_{k}$ is $\varepsilon_{1}=0.219$.

Thus, the spectrum of critical buckling forces of unbounded continuous beam with equal spans consists of the bands with continuous spectrum and with lower boundaries described by the expression $\sqrt{\lambda_{k}}=(2 k+1) \pi$, $k=1,2, \ldots$. The upper boundaries are determined by the roots of equation $\tan (\sqrt{\lambda} / 2)=\sqrt{\lambda} / 2$, having asymptotic representation $\sqrt{\lambda_{k}}=(2 k+1) \pi-2 \varepsilon_{k}$ $(k=1,2, \ldots)$. Note that the distance between the boundaries of the neighboring bands $\Delta \lambda_{k}=2 \varepsilon_{k}=4 /(2 k+1) \pi$ tends to zero when $k$ is increased.

\section{Free Vibrations of Elastic Band Lying on Periodically Distributed Rigid Supports}

Let us analyze the formation of frequency spectrum of the free transverse vibrations of the elastic band (Fig. 3) simply supported along unbounded edges $(-\infty<x<\infty$, 
$y=0, b)$ and lying on the system of rigid supports $\left(0 \leq y \leq b, x_{j}=j a, j=0, \pm 1, \pm 2, \ldots\right)$. The band is characterized by the density $\rho$, thickness $h$, width $b$, bending rigidity $D$ while the transport system is distributed with the spans $a$. The transverse vibrations are described by displacement function $w(x, y)$ that is periodic along the edges and admit the shift $x \rightarrow x+j a$. The considered spectral boundary-value problem is translation-invariant and, in correspondence with the general representation, the unknown function is found out in the form

$$
w(x+a, y) \equiv e^{i \alpha a} w(x, y), \quad 0 \leq \alpha a \leq 2 \pi .
$$

Boundary-value problem is formulated at the elementary cell $\Omega_{0}: 0 \leq x \leq a, \quad 0 \leq y \leq b$ as

$$
\begin{aligned}
& D\left(\frac{\partial^{4} w}{\partial x^{4}}+2 \frac{\partial^{4} w}{\partial x^{2} \partial y^{2}}+\frac{\partial^{4} w}{\partial y^{4}}\right)=\rho h \omega^{2} w, \\
& (x, y) \in \Omega_{0} \\
& w(x, 0)=w(x, b)=0, \\
& \frac{\partial^{2} w}{\partial y^{2}}(x, 0)=\frac{\partial^{2} w}{\partial y^{2}}(x, b)=0, \\
& 0 \leq x \leq a ; \\
& w(0, y)=w(a, y)=0, \\
& \frac{\partial w}{\partial x}(a, y)=e^{i \alpha a} \frac{\partial w}{\partial x}(0, y), \\
& \frac{\partial^{2} w}{\partial x^{2}}(a, y)=e^{i \alpha a} \frac{\partial^{2} w}{\partial x^{2}}(0, y), \\
& 0 \leq y \leq b .
\end{aligned}
$$

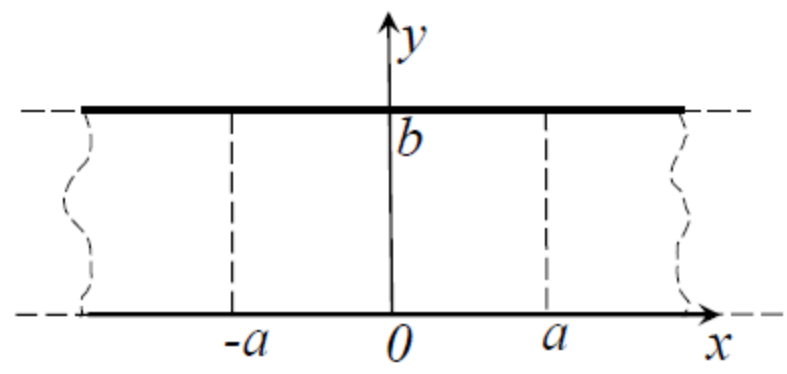

We will find out the solution of the boundary-value problem (30) in the form satisfying the simply supported boundary conditions along the band edges

$$
w(x, y)=\sum_{m=1}^{\infty} W_{m}(x) \sin \left(\frac{m \pi}{b} y\right) .
$$

Substitution of the representation (31) into the relations (30) leads to the following boundary-value problems for the functions $W_{m}(x)$ :

$$
\begin{aligned}
& \frac{d^{4} W_{m}(x)}{d x^{4}}-2 \beta_{m}^{2} \frac{d^{2} W_{m}(x)}{d x^{2}}+ \\
& +\left(\beta_{m}^{4}-\tilde{\omega}^{2}\right) W_{m}(x)=0, \\
& W_{m}(0)=W_{m}(a)=0, \quad m=1,2, \ldots, \\
& \frac{d W_{m}}{d x}(a)=e^{i \alpha a} \frac{d W_{m}}{d x}(0), \\
& \frac{d^{2} W_{m}}{d x^{2}}(a)=e^{i \alpha a} \frac{d^{2} W_{m}}{d x^{2}}(0), \\
& \beta_{m}=m \pi / b, \quad \tilde{\omega}^{2}=\rho h \omega^{2} / D .
\end{aligned}
$$

Then, the general solution of the ordinary differential equation (32) with unknown coefficients $c_{k}(k=1, \ldots, 4)$

$$
\begin{aligned}
& W_{m}(x)=c_{1} \cosh \left(\kappa_{1} x\right)+c_{2} \sinh \left(\kappa_{1} x\right)+ \\
& +c_{3} \cos \left(\kappa_{2} x\right)+c_{4} \sin \left(\kappa_{2} x\right), \\
& \kappa_{1}=\sqrt{\tilde{\omega}+\beta_{m}^{2}}, \quad \kappa_{2}=\sqrt{\tilde{\omega}-\beta_{m}^{2}}>0
\end{aligned}
$$

is subjected to the boundary conditions in (32). As a result of elementary operations, we derive the equation for $0<\alpha a<2 \pi$

$$
\begin{aligned}
& \cos \alpha a=\frac{R_{1}}{R_{2}} \equiv f, \\
& R_{1}=\kappa_{2} \sinh \left(\kappa_{1} a\right) \cos \left(\kappa_{2} a\right)- \\
& -\kappa_{1} \cosh \left(\kappa_{1} a\right) \sin \left(\kappa_{2} a\right), \\
& R_{2}=\kappa_{2} \sinh \left(\kappa_{1} a\right)-\kappa_{1} \sin \left(\kappa_{2} a\right) .
\end{aligned}
$$

Since $|\cos \alpha a|<1$ then the free vibrations are realized with the frequencies, for which $\left|f\left(\tilde{\omega}_{m}\right)\right|<1$. In particular, for asymptotic frequency values

$$
\tilde{\omega} \approx \beta_{m}^{2}, \kappa_{1} \approx \sqrt{2} \beta_{m}, \kappa_{2} \approx 0,
$$

we will have

Fig. 3. Band on rigid supports. 


$$
\begin{aligned}
& |f| \approx\left|\frac{P_{1}}{P_{2}}\right|<1, \\
& P_{1}=\sinh \left(\sqrt{2} \frac{m \pi}{b} a\right)- \\
& -\sqrt{2} \frac{m \pi}{b} a \cosh \left(\sqrt{2} \frac{m \pi}{b} a\right) \\
& P_{2}=\sinh \left(\sqrt{2} \frac{m \pi}{b} a\right)-\sqrt{2} \frac{m \pi}{b} a .
\end{aligned}
$$

Thus, the free band vibrations with frequencies $\tilde{\omega}_{m}=\beta_{m}^{2}$ are admissible.

The boundaries of bands with continuous frequency spectrum are determined with the help of conditions $\left|f\left(\tilde{\omega}_{m}\right)\right|=1$. We will have

$$
\begin{aligned}
& \left|f\left(\tilde{\omega}_{m}\right)\right|=-1: \quad \cos \left(\frac{\kappa_{2} a}{2}\right)=0, \\
& \tan \left(\frac{\kappa_{2} a}{2}\right)=\frac{\kappa_{2}}{\kappa_{1}} \tanh \left(\frac{\kappa_{1} a}{2}\right) ; \\
& \left|f\left(\tilde{\omega}_{m}\right)\right|=1: \sin \left(\frac{\kappa_{2} a}{2}\right)=0, \\
& \tan \left(\frac{\kappa_{2} a}{2}\right)=-\frac{\kappa_{1}}{\kappa_{2}} \tanh \left(\frac{\kappa_{1} a}{2}\right) .
\end{aligned}
$$

The total spectrum of free vibrations is the union of the separate spectrums, which consist of the bands with continuous frequency distributions (see the relations (33) (35) with $m=1,2,3, \ldots)$. Note here that the spectrum of free transverse vibrations of the band with simple supports along the edges $(-\infty<x<\infty, y=0, b)$ and without intermediate supports at $x=k a, k=0, \pm 1, \pm 2, \ldots$ is continuous with the lower boundary $\omega_{1}=\rho h \pi^{2} / b^{2} D$.

\section{CONCLUSION}

Analytical studies of the periodic translation-invariant mechanical systems are very important from both theoretical and practical points of view and have attracted the attention of many researchers working in the domain of theoretical and applied mechanics. Of particular interest has been the problems of elastic stability and harmonic vibration of unbounded systems.

In the paper, we considered the fundamental questions of spectral analysis of translation-invariant systems and developed the convenient representations, effective spectrum decomposition and definition of the special invariance of the problem operators. Special attention was devoted to the important examples of harmonic vibrations and stability of unbounded translation-invariant mechanical systems. Clear explanation of spectrum formation was presented.

\section{APPENDIX}

Note 1

Suppose that the considered boundary-value problem $A w(q, x)=\lambda B w(q, x)$ is defined on the finite interval $0 \leq x \leq L$ and, at the ends of interval, function $w(q, x)$ satisfies the periodicity conditions, i.e. $w(q, x) \equiv w(q, L+x)$. Assume that $L / a \square 1$ and $n=L / a \square 1$. Consequently,

$$
\alpha_{k+1}-\alpha_{k}=\frac{2 \pi}{n} k-\frac{2 \pi}{n}(k-1)=\frac{2 \pi a}{L} \square 1 .
$$

Thus, the values $\alpha_{k}$ are quasi-continuously varied and, in the limit, these values fill the continuous interval. In the asymptotical case, when $L / a \rightarrow \infty$, we arrive the representation

$$
\begin{aligned}
& w_{\alpha}(q, x)=e^{i \alpha x} w_{\alpha}^{0}(q, x), \\
& w_{\alpha}^{0}(q, x+a) \equiv w_{\alpha}^{0}(q, x) .
\end{aligned}
$$

Here we use the notation $\alpha=\alpha_{k} / a(0 \leq \alpha a \leq 2 \pi)$. This representation of eigenfunctions of translation-invariant systems is correct also for cyclic problems. It can be shown, that in the case satisfying the condition $L / a \square 1$, the interval with continuous spectrum are transformed into the "condensed zones" of eigenvalues.

Appendixes, if needed, appear before the acknowledgment.

\section{Note 2}

As it was mentioned before, the obtained general relations of translation-invariant system are correct also for cyclic systems. The corresponding relations of cyclic systems are derived from early obtained relations of translation-invariant systems with the help of replacements the coordinate $x$ by the angle $\varphi$ and the shift parameter $a$ by the period of rotation.

\section{ACKNOWLEDGMENT}

This study is partially supported by the Ministry of Science and Higher Education within the framework of the Russian State Assignment under contract No AAAA-A20120011690132-4 and partially supported by RFBR Grant 2008-00082a. 


\section{REFERENCES}

[1] Banichuk, N., Jeronen, J., Neittaanmäki, P., Saksa, T., Tuovinen, T. 2014. Mechanics of Moving Materials, Berlin/Heidelberg: Springer.

[2] Banichuk, N., Barsuk, A., Jeronen, J., Tuovinen, T. Neittaanmäki, P. 2020. Stability of Axially Moving Materials, Berlin/Heidelberg: Springer.

[3] Yakubovich, V.A., Starjinsky, V.M. 1972. Linear Differential Equations with Periodic Coefficients and Their Applications, Moscow: Nauka (in Russian).

[4] Bolotin, V.V. 1963. Nonconservative Problem of the Theory of the Elastic Stability, New York: Pergamon Press.

[5] Banichuk, N., Ivanova, S., Jeronen, J. "Moving web and dynamical problem of aerothermoelastic vibrations and instability," D.A. Indeitsev and A.M Krivtsov (Eds.): APM 2019., LNME, pp.66-71, Springer Nature Switzerland AG. 2020.

[6] Kulachenko, A., Gradin, P., Koivurova, H. "Modiling the dynamical behaviour of a paper web. Parts I,II," Computers and Structures, vol. 85, pp. 151-157, 2007.

[7] Perkins, N.C. "Linear dynamics of a translating string on an elastic foundation," Journal of Vibration and Acoustic, vol. 112, no. 2, pp. 2-7, 1990.

[8] Parker, R.G. "On the eigenvalues and critical speed stability of gyroscopic continua," ASME Journal of Applied Mechanics, vol. 65, pp. 134-140, 1998.

[9] Simpson, A. "Transverse modes and frequencies of beams translating between fixed end supports," Journal of Mechanical Engineering Science, vol. 15, pp. 159-164, 1973.

[10] Wang, Y., Huang, L., and Liu, X. "Eigenvalue and stability analysis for transverse vibrations of axially moving strings based on Haniltonian dynamics," Acta Mechanica Sinica, vol. 21, pp. 485-494, 2005.

[11] Banichuk, N., Jeronen, J., Neittaanmäki, P., and Tuovinen, T. "Static instability analysis for travelling membranes and plates interacting with axially moving ideal fluid," Journal of Fluids and Structures, vol. 26, no. 2, pp. 274-291, 2010.

[12] Kronig, R. de L., Penney, W.G. 1931. Quantum mechanics of electrons in crystal lattices, London: Proc. Roy-Soc., vol. 130 A.

\section{Creative Commons Attribution License 4.0 (Attribution 4.0 International, CC BY 4.0)}

This article is published under the terms of the Creative Commons Attribution License 4.0

https://creativecommons.org/licenses/by/4.0/deed.en_US 